\title{
Perfil epidemiológico de casos de HIV-1 atendidos em um serviço de atenção secundária em Belém-PA no período de janeiro a abril de 2012
}

\author{
Epidemiological profile of HIV-1 served on a secundary service \\ attention in Belém-PA from january to april 2012
}

\author{
Alôma Cecília Carvalho', Diana da Silva Amaral', Emanuele Cordeiro Chaves², \\ Mônica Custódia do Couto Abreu Pamplona ${ }^{1}$
}

\begin{abstract}
Resumo Objetivo: descrever o perfil epidemiológico dos casos de HIV-1 atendidos em um serviço de atenção secundária. Método: trata-se de um estudo epidemiológico, transversal, retrospectivo, documental, quantitativo, em prontuários de portadores de HIV-1 diagnosticados no período de janeiro a abril de 2012 em uma Unidade de Referência em doenças infecciosas e parasitárias em Belém-PA, correspondendo a uma amostra de 153 prontuários. Resultados: predominou o gênero masculino (60,78\%), faixa etária entre 21 a 30 anos (62,10\%), estado civil solteiro (60,13\%), escolaridade ensino fundamental (45,10\%), exposição sexual $(90,20 \%)$, orientação heterossexual $(66,01 \%)$ e não utilização de preservativo nas relações sexuais antes da infecção (75,16\%). Conclusão: houve predominância do gênero masculino, cenário que se aproxima do nacional, com uma população relativamente jovem, o que aponta vulnerabilidade desta faixa etária, solteiros; pardos, com nível de escolaridade predominante no Ensino Fundamental, o que pode interferir na falta de conhecimento e conscientização da gravidade da doença. A heterossexualização da doença aponta para um novo cenário da transmissão do HIV na região, fato que aponta a necessidade de mudanças no perfil de campanhas de prevenção da infecção. A via sexual continua apresentando-se como a principal via de exposição ao HIV, devido a não utilização do preservativo.
\end{abstract}

Descritores: doenças transmissíveis; HIV; síndrome de imunodeficiência adquirida; perfil de saúde.

Summary Purpose: To describe the epidemiology of the HIV-1 treated at a secondary care service in Belém-PA. Method: This is a cross-sectional, retrospective, documental, quantitative study of HIV-1 patients diagnosed from January to April 2012 at a Reference Unit on infectious and parasitic diseases in Belém-PA, corresponding to a sample of 153 medical records. Results: males predominated (60.78\%), aged between $21-30$ years $(62.10 \%)$, unmarried $(60.13 \%)$, primary school education $(45.10 \%)$, sexual exposure $(90.20 \%)$, heterosexual $(66.01 \%)$ and not using condoms during sexual intercourse before infection $(75.16 \%)$. Conclusion: there was a predominance of the masculine gender, a scenario that approaches the national level, with a relatively young population, which points out vulnerability of this age group, singles; pardos, with a predominance of primary schooling, which may interfere in the lack of knowledge and awareness of the severity of the disease. The heterosexualization of the disease points to a new scenario of HIV transmission in the region, a fact that points to the need for changes in the profile of infection prevention campaigns. Sexuality continues to be the main route of exposure to HIV due to non-use of condoms.

Keywords: communicable diseases; HIV; acquired immunodeficiency syndrome; health profile. 


\section{Introdução}

Na última década, verificou-se grande progresso na resposta ao vírus da imunodeficiência humana (HIV), de forma que a síndrome da imunodeficiência humana (AIDS), inicialmente considerada uma doença aguda e fatal, atualmente, apresenta perfil de doença crônica, o que se deve principalmente à instituição da terapia antirretroviral (TARV) ${ }^{1}$.

O relatório publicado em 2017 pelo Programa Conjunto das Nações Unidas sobre HIV / AIDS (UNAIDS) aponta que pela primeira vez mais da metade de todas as pessoas que vivem com HIV no mundo (53\%) tem acesso ao tratamento, e que desde 2005, o número de óbitos relacionados à AIDS reduziu quase pela metade. O cenário mundial está mais próximo à eliminação de infecções por HIV em crianças, mais pessoas vivendo com o HIV conhecem seu estado sorológico e têm acesso ao tratamento de HIV².

No Brasil, a propagação da infecção pelo HIV no país tem sofrido alterações significativas em seu perfil epidemiológico, antes restrito a alguns círculos cosmopolitas do eixo Rio - São Paulo, e predominantemente masculina, em indivíduos homossexuais e hemofílicos, a doença atualmente vivencia o processo de heterossexualização, feminização, interiorização e pauperização ${ }^{3}$. No Brasil, no período de 2007 a junho de 2017 foram notificados 194.217 casos de infecção pelo HIV, sendo 49,7\% concentrados na região Sudeste, e somente no ano de 2016 foram notificados 37.884 casos $^{4}$.

O agente causador da AIDS é o HIV, vírus que exibe uma variedade de efeitos imunológicos, dos quais o mais devastador consiste na perda completa da imunidade celular. Em consequência, as infecções oportunistas catastróficas são praticamente inevitáveis. Contudo, com o avanço das pesquisas, o tempo médio de vida dos portadores da doença e a qualidade de vida dos mesmos têm aumentado de forma significativa. Há alguns anos o diagnóstico constituía um verdadeiro decreto de morte, entretanto atualmente são verificados casos em que os portadores do vírus passam anos sem sequer desenvolver a doença ${ }^{5}$.

Há dois sorotipos de HIV, o HIV-1 é o principal sorotipo em todo o mundo, enquanto que o HIV-2 ocorre mais vulgarmente na África Ocidental. Ambos causam AIDS e os canais de transmissão são os mesmos. No entanto, a transmissão de HIV-2 é ligeiramente mais difícil e o mesmo causa uma progressão mais lenta das infecções relacionadas com o HIV e com a AIDS 6 . São conhecidos até o momento três meios de transmissão do HIV: via sexual (esperma e secreção vaginal), pelo sangue (via parenteral e vertical) e através do leite materno ${ }^{7}$.

Os resultados almejados com o presente estudo poderão auxiliar o serviço de saúde através do conhecimento do perfil epidemiológico dos indivíduos atendidos, e desta forma planejar ações de prevenção, acolhimento, aconselhamento e assistência adequada e humanizada aos portadores do vírus e aos seus parceiros sexuais, como também aos pacientes em estágios mais avançados da doença.

Desta forma, o objetivo deste estudo consiste em descrever o perfil epidemiológico dos casos de HIV-1 atendidos em um serviço de atenção secundária em Belém-PA.

\section{Método}

Trata-se de um estudo epidemiológico, transversal, retrospectivo, documental, quantitativo em prontuários de portadores de HIV-1.

A pesquisa foi desenvolvida na Unidade de Referência Especializada em Doenças Infecciosas e Parasitárias Especiais (URE-DIPE), a qual presta assistência ambulatorial aos portadores do HIV / AIDS do Estado do Pará, sendo uma Unidade de diagnóstico, tratamento e acompanhamento destas pessoas, também desenvolvendo atividades educativas e informativas para a população.

A coleta de dados foi realizada a partir de prontuários de pacientes diagnosticados com HIV-1 no período de janeiro a abril de 2012, o que correspondeu a uma amostra de 153 prontuários.

O instrumento de coleta de dados foi elaborado a partir da ficha epidemiológica criada pelo serviço da URE-DIPE e ficha do Centro de Testagem e Aconselhamento- C.T.A desenvolvida pelo Ministério da Saúde.

Para análise dos dados foi utilizado o sistema de gerenciamento de dados relacionais - SGBDR (MySQL 5.0), o qual conteve a base de dados para a pesquisa a fim de gerar as informações necessárias.

A pesquisa foi aprovada pelo Comitê de Ética em Pesquisa do Curso de Graduação em Enfermagem da Universidade do Estado do Pará, conforme Parecer n ${ }^{\circ} 205.610$ e obedeceu à Resolução 196/96 do Conselho Nacional de Saúde, vigente no período do estudo. 


\section{Resultados}

Em relação às características sociodemográficas verificou-se que nos casos de HIV-1 diagnosticados no período de janeiro a abril de 2012 houve predominância do gênero masculino, faixa etária entre de 21 a 30 anos, raça parda, solteiros e com ensino fundamental completo/incompleto (Tabela 1).

Quanto às características epidemiológicas e comportamentais evidenciou-se que a maioria dos indivíduos era heterossexual, a relação sexual foi o tipo de exposição predominante, os indivíduos não utilizavam preservativo antes da infecção, não eram usuários de drogas endovenosas e não tiveram alguma infecção sexualmente transmissível associada ao HIV / AIDS (Tabela 2).

Em relação ao número de parceiros, por gênero, evidenciou-se que houve predominância de, em média, três parceiros tanto para o sexo masculino quanto para o feminino (Tabela 3).

\section{Discussão}

O gênero masculino predominou, tal resultado concorda com dados nacionais que discutem a apresentação do agravo, que evidenciam que no período de 2002-2008, a razão de sexos, ou seja, a relação entre número de casos de AIDS em homens e mulheres, manteve-se em 15 casos em homens para cada 10 casos em mulheres; contudo, a partir de 2009, ocorreu redução gradual do número de casos em mulheres e aumento entre os homens, o que culminou na razão de sexos de 22 casos de AIDS em homens para cada 10 mulheres ${ }^{4}$. Estudo realizado em Belém-PA identificou ainda que 71,1\% dos pacientes com HIV-AIDS que evoluíram ao óbito eram homens ${ }^{8}$.

Tabela 1. Características sociodemográficas dos participantes do estudo, Belém/PA, Brasil (n=153)

\begin{tabular}{|c|c|c|}
\hline Variáveis & $\mathbf{n}^{*}$ & $f(\%)^{* *}$ \\
\hline \multicolumn{3}{|l|}{ Gênero } \\
\hline Masculino & 93 & 60,78 \\
\hline Feminino & 60 & 39,22 \\
\hline \multicolumn{3}{|l|}{ Faixa etária (anos) } \\
\hline 18 a 20 & 5 & 3,27 \\
\hline 21 a 30 & 95 & 62,10 \\
\hline 31 a 40 & 34 & 22,22 \\
\hline 41 a 59 & 18 & 11,76 \\
\hline 60 anos & 1 & 0,65 \\
\hline \multicolumn{3}{|l|}{ Raça } \\
\hline Branca & 12 & 7,84 \\
\hline Parda & 133 & 86,93 \\
\hline Negra & 8 & 5,23 \\
\hline \multicolumn{3}{|l|}{ Estado civil } \\
\hline Casado/Relação Estável & 49 & 32,03 \\
\hline Solteiro & 92 & 60,13 \\
\hline Viúvo & 5 & 3,27 \\
\hline Outros & 7 & 4,57 \\
\hline \multicolumn{3}{|l|}{ Escolaridade } \\
\hline Analfabeto & 5 & 3,27 \\
\hline Fundamental completo/incompleto & 69 & 45,10 \\
\hline Médio completo/incompleto & 62 & 40,52 \\
\hline Superior & 17 & 11,11 \\
\hline
\end{tabular}

*n: representação bruta por categoria de estudo; **f (\%): representação proporcional por categoria de estudo. Fonte: Protocolo de pesquisa. 
Tabela 2. Características epidemiológicas e comportamentais dos portadores de HIV-1 participantes do estudo, Belém/PA, Brasil $(\mathrm{n}=153)$
Variáveis
$\mathbf{n}^{*}$
$f(\%)^{* *}$

Tipo de exposição

$\begin{array}{lrr}\text { Relação sexual } & 138 & 90,20 \\ \text { Relação sexual e transfusão sanguínea } & 8 & 5,23 \\ \text { Relação sexual e compartilhamento de seringas } & 5 & 3,27 \\ \text { Compartilhamento de seringas } & 2 & 1,30\end{array}$

Orientação sexual

$\begin{array}{lrr}\text { Heterossexual } & 101 & 66,01 \\ \text { Homossexual } & 43 & 28,11 \\ \text { Bissexual } & 9 & 5,88\end{array}$

Uso de preservativo antes da infecção

$\begin{array}{lrr}\text { Sim } & 38 & 24,84 \\ \text { Não } & 115 & 75,16\end{array}$

Usuário de drogas endovenosas

$\begin{array}{lrr}\text { Sim } & 6 & 3,92 \\ \text { Não } & 147 & 96,08\end{array}$

Infecção Sexualmente Transmissível (IST) associada ao HIV / AIDS

$\begin{array}{lrr}\text { Sim } & 35 & 22,88 \\ \text { Não } & 118 & 77,12\end{array}$

Tipo de IST's***

\begin{tabular}{lcc} 
Candidíase & 1 & 2,86 \\
Gonorréia & 1 & 2,86 \\
Herpes genital & 3 & 8,57 \\
HPV & 1 & 2,86 \\
Sífilis & 6 & 17,14 \\
Não informado & 23 & 65,71 \\
\hline
\end{tabular}

*n: representação bruta por categoria de estudo; **f $(\%)$ : representação proporcional por categoria de estudo; ***Considerando apenas os casos que apresentaram IST associada ao HIV/AIDS (N=35). Fonte: Protocolo de pesquisa.

Tabela 3. Número médio de parceiros, por gênero dos portadores de HIV-1 participantes do estudo, Belém/PA, Brasil (n=153)

\begin{tabular}{|c|c|c|c|c|}
\hline \multirow{2}{*}{$\begin{array}{l}\text { Número médio de } \\
\text { parceiros }\end{array}$} & \multicolumn{2}{|c|}{ Masculino } & \multicolumn{2}{|c|}{ Feminino } \\
\hline & $n^{*}$ & $f(\%)^{* *}$ & $\mathrm{n}^{*}$ & $f(\%)^{* *}$ \\
\hline Três & 57 & 61,29 & 39 & 65,00 \\
\hline Cinco & 25 & 26,88 & 16 & 26,67 \\
\hline Dez & 11 & 11,83 & 5 & 8,33 \\
\hline Total & 93 & 100,00 & 60 & 100,00 \\
\hline
\end{tabular}

*n: representação bruta por categoria de estudo; **f (\%): representação proporcional por categoria de estudo. Fonte: Protocolo de pesquisa.

Tal situação pode estar relacionada à própria atividade sexual masculina, vista como um rito para a construção da virilidade, já que no Brasil o homem é representado como sujeito sexualmente ativo e desejoso de sexo, diferentemente da mulher, que é vista como passiva e cuja experiência sexual depende da formação de vínculo afetivo para que ocorra. Para o senso comum, o homem não rejeita sexo e no imaginário social o homem é visto como uma "máquina sexual", e tem que estar pronto a todo momento para atividade sexual, para que possa comprovar sua virilidade ${ }^{9}$, o que indubitavelmente o expõe a situação de maior vulnerabilidade. 
Quanto à faixa etária, verificou-se que houve predominância entre os indivíduos entre 21 a 30 anos de idade, o que concorda com os dados nacionais, tendo em vista que no período de $2007-2017,52,5 \%$ dos casos de HIV notificados se encontravam na faixa etária de 20 a 34 anos ${ }^{4}$. Tal achado reflete em fatores econômicos, afetivos e sociais, tendo em vista que se trata de uma doença de alta morbimortalidade em adultos jovens, considerados de maior produtividade econômica e sexual ${ }^{10}$.

A raça parda foi a mais frequente entre os participantes do estudo, o que vai ao encontro do que sido evidenciado acerca do agravo ${ }^{3,8,11}$, tendo em vista que a AIDS continua avançando entre as populações mais vulneráveis socioeconomicamente, o que é expresso pelo aumento persistente da proporção de casos com raça parda e redução na raça branca ${ }^{11}$.

O estado civil predominante foi solteiro, o que também foi mostrado em estudo realizado no município de São José- Santa Catarina, envolvendo portadores de HIV/AIDS atendidos a nível ambulatorial ${ }^{12}$. Os indivíduos que não mantém uma relação matrimonial são mais propensos a ter maior quantidade de parceiros sexuais e, desta forma aumentam o risco de adquirir a infecção ${ }^{13}$.

A escolaridade dos participantes do estudo foi baixa, com predominância do ensino fundamental. $O$ fato de a doença atingir cada vez mais pessoas com menor escolaridade está relacionado com o reduzido acesso às informações ${ }^{14}$, além disso, tem se verificado que quanto maior o grau de instrução mais frequente é o uso de preservativos, enquanto que quanto menor a escolaridade, maior o uso de drogas. Portanto, a baixa escolaridade constitui um fator de susceptibilidade à infecção pelo HIV ${ }^{15}$.

Nesse sentido, diversos estudos indicam que apenas aumentar o nível de informação sobre as vias de transmissão do HIV e a necessidade de se utilizar o preservativo não garante mudanças de práticas sexuais ${ }^{16}$, como pode ser observado no estudo em questão, no qual a maioria dos participantes referiram não utilizar o preservativo antes da infecção e, como consequência, a via de exposição predominante foi a sexual, o que também tem emergido em outros estudos ${ }^{17,18}$.

O número médio de parceiros, tanto para homens quanto para mulheres, foi três. Contudo, tal dado de forma isolada é insuficiente para determinar a vulnerabilidade do indivíduo, pois muitos riscos associados à experiência sexual dependem do tipo de parceiro que se tem em dado momento e a história sexual ao longo da vida ${ }^{19}$.

Com relação à orientação sexual, os indivíduos heterossexuais foram os mais acometidos pelo agravo, assim como identificado em outros estudos ${ }^{3,20,21}$. A epidemia da AIDS no Brasil é subdividida em três fases: a primeira fase inicia-se na década de 80 , caracterizada pela infecção a grupos homossexuais, bissexuais e receptores de sangue e hemoderivados; a segunda (início da década de 90) é marcada pela infecção em usuários de drogas injetáveis e aumento de casos heterossexuais; e na terceira fase, atual, houve um aumento da transmissão por indivíduos heterossexuais, culminando na efetiva contaminação em mulheres ${ }^{21}$.

Maioria expressiva dos participantes não utilizava drogas endovenosas, seguindo a tendência nacional identificada em levantamento realizado pelo Ministério da Saúde referente ao período de 2007-2017, no qual se identificou que 2,9\% dos casos de HIV notificados entre homens e 1,7\% entre mulheres eram de usuários de drogas injetáveis ${ }^{4}$, além disso, em estudo realizado em uma maternidade de Belém-PA com mulheres HIV positivas que pariram em um Hospital de Referência apenas 11,4\% das participantes relataram uso de drogas ilícitas ${ }^{22}$. $\mathrm{O}$ achado do presente estudo confirma a exposição sexual como principal via de infecção, como mencionado anteriormente.

Em relação a outras IST's nos últimos doze meses, verificou-se que a maioria informou não ter apresentado, e dentre os que disseram ter tido, a predominante foi sífilis, como também foi evidenciado em outro estudo envolvendo essa população ${ }^{23}$.

As limitações do estudo estão relacionadas à natureza transversal da pesquisa, que verificou a ocorrência dos eventos em um único momento do tempo, e por se tratar de um estudo retrospectivo e documental existe o viés de informação e a impossibilidade de esclarecimento de dúvidas diretamente com os sujeitos. Ressalta-se, desta forma, que o estudo tem natureza apenas descritiva e sem análise inferencial, impossibilitando a análise do quanto os achados podem gerar risco de adoecimento ou não.

\section{Conclusão}

A amostra investigada, referente aos pacientes diagnosticados com HIV-1 no período de janeiro a abril de 2012, foi predominantemente do gênero masculino, cenário que se aproxima do nacional, com uma população relativamente jovem, o que aponta vulnerabilidade desta faixa etária, solteiros; pardos, com nível 
de escolaridade predominante no Ensino Fundamental, o que pode interferir na falta de conhecimento e conscientização da gravidade da doença.

A heterossexualização da doença aponta para um novo cenário da transmissão do HIV em nossa região, fato que aponta a necessidade de mudanças no perfil de campanhas de prevenção da infecção. Sendo que a via sexual continua apresentando-se como a principal via de exposição ao HIV, devido a não utilização do preservativo.

Nesse sentido, faz-se necessária a realização de mais estudos na área, direcionando os mesmos para as novas formas de contaminação e público que vem sendo mais atingido, visando elucidem quais as melhores estratégias podem ser utilizadas pelos profissionais de saúde no combate à infecção, principalmente no que tange à adesão da população às medidas preventivas, atualmente negligenciadas, como o uso do preservativo.

Sensibilizar a população da gravidade da doença é necessário, assim como elucidar a importâncias de barreiras preventivas frente à contaminação pelo vírus, efetivando campanhas para prevenção do HIV.

\section{Referências}

1. Medeiros RCSC, Medeiros JA, Silva TAL, Andrade RD, Medeiros DC, Araújo JS, et al. Qualidade de vida, fatores socioeconômicos e clínicos e prática de exercício físico em pessoas vivendo com HIV/aids. Rev Saude Publica. 2017;51(66):1-8. PMid:28099550.

2. Programa Conjunto das Nações Unidas - UNAIDS [Internet]. Ending AIDS: Progress towards the 90-90-90 targets. Genebra: UNAIDS; 2017 [citado em 2017 Dez 10]. Disponível em: http://www.unaids.org/sites/default/files/media_asset/Global_AIDS_update_2017_en.pdf

3. Abreu SR, Pereira BM, Silva NM, Moura LRP, Brito CMS, Câmara JT. Estudo epidemiológico de pacientes com infecção pelo vírus da imunodeficiência humana/síndrome da imunodeficiência adquirida (HIV/aids), Caxias-MA. Revista Interdisciplinar. 2016;9(4):132-41.

4. Brasil. Ministério da Saúde [Internet]. Boletim epidemiológico: HIV AIDS 2017. Brasília: Ministério da Saúde; 2017 [citado em 2017 Dez 15]. Disponível em: http://www.aids.gov.br/ptbr/pub/2017/boletim-epidemiologico-hivaids-2017

5. Almeida EL, Araújo GBS, Santos VA, Bustorff LACV, Pereira AVL, Dias MD. Adesão dos portadores do HIV/AIDS ao tratamento: fatores intervenientes. Rev Min Enferm. 2011;15(2):208-16.

6. Veronesi R, Focaccia R. Tratado de infectologia. 4 d. São Paulo: Atheneu; 2009.

7. Soares AP. Transmissão consciente do vírus HIV: tentativa de homicídio ou lesão corporal? Blumenal: Universidade Regional de Blumenal; 2010.

8. Santos ERF, Leite GP No, Cunha RA, Bezerra MPC, Valente RFB. Perfil de pacientes HIV-AIDS que evoluíram ao óbito em um hospital de referência em Belém-PA. Rev Para Med. 2015;29(3):53-60.

9. Domingues PS, Gomes AMT, Oliveira DC. Representações sociais de homens sobre o ser homem e suas implicações para o HIV/AIDS [Men's social representations of being a man and their implications for HIV/AIDS]. Rev Enferm UERJ. 2016;24(6):1-6. http://dx.doi.org/10.12957/ reuerj.2016.8779.

10. BoffJA, Dallacosta FM. Notificações de AIDS/HIV: uma análise em um município de Santa Catarina. III Jornada Integrada em Biologia [Internet]. Joaçaba: Unoesc; 2016 [citado em 2017 Dez 16]. Disponível em: http://editora.unoesc.edu.br/index.php/jornadaintegradaembiologia/ article/view/10311

11. Fry PH, Monteiro S, Maio MC, Bastos FI, Santos RV. AIDS tem cor ou raça? Interpretação de dados e formulação de políticas de saúde no Brasil. Cad Saude Publica. 2007;23(3):497-507, discussion 508-23. http://dx.doi.org/10.1590/S0102-311X2007000300002. PMid:17334558.

12. Bertoni RF, Bunn K, Silva J, Traebert J. Perfil demográfico e socioeconômico dos portadores de HIV/AIDS do ambulatório de controle de DST/AIDS de São José, SC. AMC Arq Catarin Med. 2010;39(4):75-9.

13. Monteiro MRCC, Rodrigues MPC, Rossy MCNB, Pelaes TS, Gonçalves EAC, Turiel PRF. Infecções em pacientes com HIV/AIDS de hospital referência, em Belém. Rev Para Med. 2008;22(3):1-7.

14. Freitas JG, Galvão MTG, Araújo MFM, Costa E, Lima ICV. Enfrentamentos experimentados por homens que vivem com HIV/AIDS no ambiente de trabalho. Rev Esc Enferm USP. 2012;46(3):720-6. http://dx.doi.org/10.1590/S0080-62342012000300026. PMid:22773495.

15. Brito AM, Castilho EA, Szwarcwald CL. AIDS e infecção pelo HIV no Brasil: uma epidemia multifacetada. Rev Soc Bras Med Trop. 2001;34(2):20717. http://dx.doi.org/10.1590/S0037-86822001000200010. PMid:11391445.

16. Brasil. Ministério da Saúde. HIV/Aids, hepatites virais e outras DST. Brasília: Ministério da Saúde; 2010. (Cadernos de Atenção Básica; 8).

17. Schuelter-Trevisol F, Paolla P, Justino AZ, Pucci N, Silva ACB. Perfil epidemiológico dos pacientes com HIV atendidos no sul do estado de Santa Catarina, Brasil, em 2010. Epidemiol Serv Saude. 2013;22(1):87-94. http://dx.doi.org/10.5123/S1679-49742013000100009.

18. Silva RAR, Duarte FHS, Nelson ARC, Holanda JRR. A epidemia da AIDS no Brasil: Análise do perfil atual. Rev Enferm UFPE on line [Internet]. 2013 Out [citado em 2017 Dez 16];7(10):6039-46. Disponível em: https://periodicos.ufpe.br/revistas/revistaenfermagem/article/ viewFile/12233/14841. 
19. Aboim S. Risco e prevenção do HIV/Aids: uma perspectiva biográfica sobre os comportamentos sexuais em Portugal. Cien Saude Colet. 2012;17(1):99-112. http://dx.doi.org/10.1590/S1413-81232012000100013. PMid:22218544.

20. Silva SFR, Pereira MRP, Motta R No., Ponte MF, Ribeiro IF, Costa PFTF, et al. Aids no Brasil: uma epidemia em transformação. Rev Bras Anal Clin. 2010;42(3):209-12.

21. Soares FNS, Morais MTM. Perfil epidemiológico e sócio demográfico dos pacientes vivendo com HIV/AIDS cadastrados no município de Vitória da Conquista/BA. Rev Saúde.Com [Internet]. 2014 [citado em 2017 Dez 16];10(1):54-63. Disponível em: http://www.uesb.br/revista/ rsc/ojs/index.php/rsc/article/view/212/251

22. Araújo EC, Drosdoski FS, Nunes NB Jr, Ferreira PGM. Transmissão vertical do HIV em maternidade de referência na Amazônia Brasileira. Rev Para Med. 2015;29(2):17-21.

23. Rodrigues EHG, Abath FGC. Doenças sexualmente transmissíveis em pacientes infectados com HIV/AIDS no estado de Pernambuco, Brasil. Rev Soc Bras Med Trop. 2000;33(1):47-52. http://dx.doi.org/10.1590/S0037-86822000000100007. PMid:10881118.

\section{Autor correspondente}

Alôma Cecília Carvalho

Av. Ricardo Borges, 42, Condomínio Viver Castanheira, ap. 401, Bairro Guanabara

CEP 67110-290, Ananindeua, PA, Brasil

Tel: (91) 98187-6152

E-mail:alomaenf@hotmail.com

\section{Informação sobre os autores}

ACC, DSA são enfermeiras, egressas da Universidade do Estado do Pará, (UEPA).

ECC é enfermeira; doutoranda em Doenças Tropicais pela Universidade Federal do Pará (UFPA).

MCCAP é enfermeira; doutora em Biologia dos Agentes Infecciosos e Parasitários e Docente da Universidade do Estado do Pará (UEPA).

\section{Contribuição dos autores}

Todas as autoras participaram da concepção e desenvolvimento da pesquisa, desenho metodológico, coleta e análise dos dados, levantamento da literatura, redação do manuscrito e revisão crítica.

Todos os autores leram e aprovaram a versão final submetida ao Pará Research Medical Journal. 